\title{
The investigation and management of pancreatic exocrine insufficiency: A retrospective cohort study
}

\author{
Authors: Benjamin M Shandro, ${ }^{A}$ Jennifer Ritehnia, ${ }^{B}$ Joshua Chen, ${ }^{B}$ Rani Nagarajah $^{C}$ and Andrew Poullis ${ }^{D}$
}

\begin{abstract}
Pancreatic exocrine insufficiency (PEI) is associated with significant gastrointestinal symptoms, but is readily treated by pancreatic enzyme replacement therapy (PERT). We reviewed our current practice and examined the factors that predict repeating a positive faecal elastase-1 (FE1; $<200 \mu \mathrm{g} / \mathrm{g}$ ), the repeat FE1 being normal, initiation of PERT and clinical response to treatment. A single-centre retrospective cohort study was conducted. Outpatients with FE1 $<200 \mu \mathrm{g} / \mathrm{g}$ between 2012 and 2018 were included. Logistic regression was used to explore the associations with each outcome, with statistical adjustment for confounders. Two-hundred and ten patients were included; $28.1 \%$ of patients had their FE1 repeated, $47.5 \%$ of whom had a normal repeat result. Patients with initial FE1 $<15 \mu \mathrm{g} / \mathrm{g}$ were unlikely to be reclassified on repeat testing. Patients with a confirmatory low FE1, abnormal pancreatic imaging or abnormal nutrition blood tests were more likely to be started on PERT (all $p<0.05$ ). Patients with abnormal pancreatic imaging were 10 times more likely to respond to PERT (odds ratio 10.70; 95\% confidence interval 1.62-70.70; $\mathrm{p}=0.01$ ). Augmenting clinical judgement with pancreatic imaging and repeat FE1 testing could improve the rate of PERT prescription and inform the approach to non-response, particularly in cases where there is diagnostic doubt.
\end{abstract}

KEYWORDS: Pancreatic exocrine insufficiency, faecal elastase, chronic pancreatitis, pancreatic enzyme replacement therapy

DOI: 10.7861/clinmed.2020-0506

\section{Introduction}

Pancreatic exocrine insufficiency (PEI) occurs when there is inadequate secretion or function of pancreatic enzymes for normal digestion. PEI can result from impaired production or drainage of pancreatic enzymes, reduced entero-hormonal stimulation of the exocrine pancreas, or asynchronous digestion

Authors: Aresearch fellow, St George's University Hospitals NHS Foundation Trust, London, UK; 'B medical student, St George's, University of London, London, UK; ' dietitian, St George's University Hospitals NHS Foundation Trust, London, UK; 'D consultant gastroenterologist, St George's University Hospitals NHS Foundation Trust, London, UK due to dysmotility or abnormal anatomy. ${ }^{1}$ It is found most commonly in patients with chronic pancreatitis, pancreatic cancer, pancreatic resection or cystic fibrosis. There is also some evidence of an association with coeliac disease, previous upper gastrointestinal (GI) surgery and diabetes mellitus. ${ }^{1}$

Patients with PEI may present with chronic diarrhoea, steatorrhoea, abdominal pain, weight loss or malnutrition. These presentations are non-specific, and as a result their initial investigation and management will often be carried out by general practitioners or physicians working in non-GI specialties. ${ }^{2}$

The diagnosis of PEI has traditionally required invasive direct pancreatic function testing, or the calculation of the coefficient of faecal fat absorption (CFA) from stool collected over 72 hours. ${ }^{3}$ These time-consuming tests have largely been replaced in clinical practice by the measurement of faecal elastase-1 (FE1). An FE1 $<200 \mu \mathrm{g} / \mathrm{g}$ is considered diagnostic of PEI in accordance with the intended use label of the test, and can be further subclassified into mild-moderate PEI (100-199 $\mu \mathrm{g} / \mathrm{g})$ and severe disease $(<100 \mu \mathrm{g} / \mathrm{g})$. The FE1 assay is practical and inexpensive, requiring only a single stool sample. However, dilute stool samples can yield falsely low results. ${ }^{4}$ A recent meta-analysis demonstrated a pooled sensitivity and specificity for $\mathrm{FE} 1$ of $77 \%$ and $88 \%$, respectively, compared with direct pancreatic function tests, and $96 \%$ and $88 \%$, respectively, compared with CFA. ${ }^{3}$ Overall, FE1 is thought to misclassify approximately $10 \%$ of patients, though repeat testing is not widely practiced. ${ }^{3}$

PEI is associated with substantial morbidity, but can be readily treated by pancreatic enzyme replacement therapy (PERT). ${ }^{5,6}$ It is estimated that $80 \%$ of patients respond clinically, and PERT has been shown to relieve the symptoms of PEI and improve survival. ${ }^{6-8}$ However, studies have shown that treatment is initiated in as few as $21 \%$ of patients who might benefit, and that dosing may be inadequate in many patients on treatment. ${ }^{9,10}$ However, the factors predicting initiation of, and response to, PERT are unknown.

The timely, accurate diagnosis of PEI and initiation of effective treatment are of clear benefit to patients. Several international guidelines and reviews have been published offering recommendations on the investigation and management of patients with PEI. ${ }^{11-14}$ Where there is consensus between these publications, a current gold standard can be inferred (Table 1). We aimed to review clinical practice at our centre against this standard, and study the factors that predict repeat FE1 testing, the repeat $F E 1$ being $\geq 200 \mu \mathrm{g} / \mathrm{g}$, initiation of PERT and clinical response to treatment. 
Table 1. Concise current gold standard for the initial management of pancreatic exocrine insufficiency

FE1 should be repeated in cases of diagnostic doubt.

Patients with PEI should undergo pancreatic imaging at diagnosis.

Patients with PEI should undergo biochemical screening for malnutrition.

Patients with PEI should be prescribed PERT, at an initial dose of $\geq 40,000 \mathrm{IU} / \mathrm{meal}$.

\author{
Alcohol and smoking cessation should be \\ advised. \\ Patients with PEI should be referred to a \\ dietitian. \\ Response to PERT should be monitored at \\ follow-up.
}

FE1 has a false positive rate of approximately $10 \% .{ }^{4}$ A low FE1 should be repeated in patients with a low pre-test probability of PEI, such as those lacking established risk factors. ${ }^{7,13}$

This provides structural information about the pancreas and excludes pancreatic cancer as a potential aetiology. ${ }^{11-14}$

Malnutrition is common in patients with PEI, and responsible for significant morbidity if left untreated eg osteoporosis. ${ }^{11-13}$

PERT is the cornerstone of PEI treatment. There is evidence that previous recommendations to start PERT at a dose of $20,000 \mathrm{IU} / \mathrm{meal}$ undertreats twothirds of patients, therefore 40,000-50,000 IU/meal is now preferred. ${ }^{12-15}$

Both alcohol and smoking are considered risk factors for the progression of PEI, particularly in patients with chronic pancreatitis. ${ }^{11-13}$

Dietary management is an important aspect of treatment. Dietitians are expert in assessing malnutrition, obtaining diet histories, and tailoring meal content and PERT regimens to individual circumstances. ${ }^{11-13}$

Clinical response is a satisfactory outcome in most settings. ${ }^{12}$ Where available, the CFA or 13C-mixed triglyceride breath test can be used to identify patients with symptomatic improvement who remain at risk of malnutrition. ${ }^{15}$

CFA $=$ coefficient of faecal fat absorption; FE1 = faecal elastase-1; PEI = pancreatic exocrine insufficiency; PERT = pancreatic enzyme replacement therapy

\section{Methods}

A retrospective cohort study was conducted at St George's University Hospitals NHS Foundation Trust. Adult outpatients tested for PEI with the FE1 assay between 01 September 2012 and 31 August 2018 were identified from the biochemistry database. Patients with a positive FE1 result ( $<200 \mu \mathrm{g} / \mathrm{g}$ ) were included in the study. FE1 was measured using a sandwich enzyme-linked immune-absorbent assay (ELISA) with two monoclonal antibodies highly specific for human pancreatic elastase-1 (ScheBo Biotech, Giessen, Germany). Patients already taking PERT at the time of FE1 testing were excluded.

Demographic and clinical information were retrieved from the electronic patient record. Variables of interest and statistical methods were selected in advance. We collected data on age, sex, ethnicity, presenting symptoms and relevant comorbidities: chronic pancreatitis, pancreatic cancer, previous upper GI surgery, diabetes mellitus, alcohol excess (classified as never, previous or current excess; with excess defined as $>14$ units per week) and smoking history (classified as never smoked, ex-smoker or current smoker). ${ }^{16}$ Comorbidities were defined by clinical documentation preceding the FE1 test, to reflect what would have been available to the requesting clinician.

We noted the number of patients whose FE1 test had been repeated, and the result of the repeat test. Patients with a low FE1 not followed by a normal result were considered to have PEI. In patients with $\mathrm{PEI}$, we noted the number in whom pancreatic imaging and nutritional blood tests (defined as $\geq 3$ of serum ferritin, folate, vitamin B12, vitamin D, magnesium and albumin) had been performed in the 6 months before or after FE1 testing. We also noted the number of patients prescribed PERT, the initial dose, those referred for formal dietetic assessment, offered followup and the clinical response to PERT (defined by subjective change in symptoms).

Summative statistics were used to describe the study population. Logistic regression was used to explore the factors associated with repeat FE1 testing, the repeat FE1 being $\geq 200 \mu \mathrm{g} / \mathrm{g}$, the initiation of PERT and clinical response. Multivariable logistic regression was used to adjust each estimate for age, sex and ethnicity. Complete case analysis was used where any data were missing. All statistical analyses were performed using Stata version 13.1 (StataCorp, College Station, USA). This study was approved by the Health Research Authority (HRA) and Health and Care Research Wales (HCRW; reference number: 20/LO/0433).

\section{Results}

One-thousand and thirty-four outpatients were tested for PEI using the FE1 assay during the study period. Two-hundred and seventeen patients had an FE1 result $<200 \mu \mathrm{g} / \mathrm{g}$, seven of whom were excluded as already taking PERT. Two-hundred and ten patients were included in the final analysis; mean age was 56 years (standard deviation (SD) 16.3) and 111 (52.9\%) were male. 13.3\% of FE1 assays were requested by non-gastroenterologists. Detailed tables of results can be found in supplementary material S1.

\section{Repeat FE1 testing}

Fifty-nine patients with a low FE1 (28.1\%) underwent repeat FE1 testing. The univariable analysis, adjusted for age, sex and ethnicity, showed few differences between patients who did and did not undergo repeat testing. The initial FE1 value was not associated with whether or not the test was repeated (odds ratio (OR) 1.00; 95\% confidence interval (CI) 1.00-1.00). Patients presenting with bloating and flatulence were more likely to undergo repeat testing than those without (OR 3.09; 95\% CI 1.51-6.29; and OR 7.00; 95\% CI 1.89-25.90), and patients of Asian ethnicity and current smokers were less likely to undergo repeat FE1 testing (OR 0.38; 95\% CI 0.15-0.94; and OR 0.29; 95\% CI 0.11-0.76).

Of the patients who underwent repeat FE1 testing, 28 (47.5\%) had a normal FE1 ( $\geq 200 \mu \mathrm{g} / \mathrm{g}$ ) on the second test. The adjusted 
univariable analysis showed that for every $1 \mu \mathrm{g} / \mathrm{g}$ increase in FE1, there was a $1 \%$ increase in the odds of having a normal FE1 on repeat testing (OR 1.01; 95\% CI 1.00-1.02). Abdominal pain was the only other factor associated with having a repeat FE1 $\geq 200$ $\mu \mathrm{g} / \mathrm{g}$ (OR 4.97; 95\% CI 1.13-21.76). No patients with pancreatic cancer underwent repeat testing, therefore logistic regression could not be performed due to complete separation.

Of the 28 patients with normal FE1 values on repeat testing, 17 $(60.7 \%)$ were subsequently diagnosed with functional GI disease, four $(14.3 \%)$ with bile acid malabsorption, two (7.1\%) with Crohn's disease, one $(3.6 \%)$ with untreated coeliac disease and one $(3.6 \%)$ with idiopathic intestinal lymphangiectasia. Three patients $(10.7 \%)$ had known chronic pancreatitis, and in two of these cases the repeat FE1 result was considered a false negative by the treating clinician.

\section{Initial management of PEI}

Once repeat FE1 test results had been taken into account, 182 patients $(86.7 \%)$ with a low FE1 had a final diagnosis of PEI. Of these, $72(39.6 \%)$ had mild-moderate PEI (FE1 100-199 $\mu \mathrm{g} / \mathrm{g}$ ) and $110(60.4 \%)$ had severe disease (FE1 $<100 \mu \mathrm{g} / \mathrm{g})$. Of patients with PEI, $83.5 \%$ had one or more relevant comorbidity. The PEI cohort characteristics are shown in Table 2.

One-hundred and forty-nine patients with PEI (81.9\%) underwent cross-sectional imaging in the 6 months before and after FE1 testing. Imaging modalities included ultrasound (23.5\%), computed tomography $(60.4 \%)$, magnetic resonance imaging $(15.4 \%)$, and endoscopic ultrasound $(0.7 \%)$. Inadequate views of the pancreas were reported in $17.1 \%$ of ultrasounds. Of patients with adequate imaging, 67 (46.9\%) had pancreatic abnormalities. Thirty-six patients $(25.2 \%$ ) were found to have radiological evidence of chronic pancreatitis, and seven patients (4.9\%) were diagnosed with pancreatobiliary cancer. Eighteen cases (47.4\%) of chronic pancreatitis and two pancreatobiliary cancers $(28.6 \%)$ were diagnosed radiologically after the positive FE1 result. The other pancreatic abnormalities detected on imaging were 11 cases $(7.7 \%)$ of pancreatic atrophy; nine $(6.3 \%)$ of fatty pancreas; one $(0.7 \%)$ acute pancreatitis; one $(0.7 \%)$ autoimmune pancreatitis; and two patients (1.4\%) with post-surgical anatomy.

One-hundred and twenty-four patients with PEI (68.1\%) had nutrition blood tests sent, 65 (52.4\%) of whom had one or more abnormal result. Of patients with PEI tested for each nutritional parameter, we detected ferritin $<30 \mu \mathrm{g} / \mathrm{L}$ in $12.9 \%$, folate $<5 \mathrm{ng} / \mathrm{L}$ in $16.7 \%$, vitamin $\mathrm{B} 12<180 \mathrm{pg} / \mathrm{mL}$ in $2.3 \%$, vitamin $\mathrm{D}<30 \mathrm{ng} / \mathrm{mL}$ in $24.1 \%$, magnesium $<0.7 \mathrm{nmol} / \mathrm{L}$ in $23.8 \%$ and albumin $<35 \mathrm{~g} / \mathrm{L}$ in $25.8 \%$.

One-hundred and three patients (56.6\%) with PEI were treated with PERT. The median starting dose of PERT was $50,000 \mathrm{IU} / \mathrm{meal}$ (interquartile range 0-0; range 10,000-100,000). Seventy-three treated patients $(70.9 \%)$ were referred for dietetic assessment and $89(86.4 \%)$ were offered follow-up. However, only four untreated patients $(5.1 \%)$ were referred to the dietitian and $10(12.7 \%)$ offered follow-up.

Table 2. Presenting symptoms and comorbidities in patients with pancreatic exocrine insufficiency, and results of adjusted univariable analysis of their association with initiation of PERT and clinical response

\begin{tabular}{|c|c|c|c|}
\hline Variable & $\begin{array}{l}\text { Patients with PEI, } \\
n=182, n(\%)\end{array}$ & $\begin{array}{l}\text { Association with initiation } \\
\text { of PERT, OR }(95 \% \mathrm{CI})^{a}\end{array}$ & $\begin{array}{l}\text { Association with clinical response } \\
\text { to PERT, OR }(95 \% \mathrm{CI})^{a}\end{array}$ \\
\hline \multicolumn{4}{|l|}{ Presenting symptoms } \\
\hline Abdominal pain & $91(50.0)$ & $1.30(0.70-2.40)$ & $1.01(0.29-3.50)$ \\
\hline Diarrhoea & $126(69.2)$ & $0.78(0.40-1.49)$ & $0.79(0.21-3.00)$ \\
\hline Weight loss & $84(46.2)$ & $1.60(0.85-3.01)$ & $6.83(1.23-37.93)^{b}$ \\
\hline Steatorrhoea & $31(17.0)$ & $2.54(1.06-6.09)^{b}$ & $2.94(0.57-15.23)$ \\
\hline Bloating & $37(20.3)$ & $1.11(0.52-2.36)$ & $0.47(0.13-1.75)$ \\
\hline Flatulence & $6(3.3)$ & $0.30(0.05-1.74)$ & $0.04(0.002-0.93)^{b}$ \\
\hline \multicolumn{4}{|l|}{ Comorbidities } \\
\hline Chronic pancreatitis & $21(11.5)$ & $4.13(1.31-13.02)^{b}$ & $3.06(0.34-27.66)$ \\
\hline Pancreatic cancer & $5(2.75)$ & $3.97(0.42-37.29)$ & $\mathrm{n} / \mathrm{a}$ \\
\hline Upper GI surgery & $16(8.79)$ & $1.37(0.47-4.04)$ & $0.22(0.04-1.16)$ \\
\hline Diabetes mellitus & $64(35.2)$ & $2.21(1.11-4.42)^{b}$ & $0.54(0.14-2.07)$ \\
\hline \multicolumn{4}{|l|}{ Alcohol excess } \\
\hline Previous & $25(18.5)$ & $3.18(1.04-9.71)^{b}$ & $0.68(0.11-4.34)$ \\
\hline Current & $27(20.0)$ & $1.47(0.56-3.88)$ & $4.52(0.36-56.73)$ \\
\hline \multicolumn{4}{|l|}{ Smoking } \\
\hline Previous & $24(18.3)$ & $0.73(0.27-2.01)$ & $4.39(0.19-102.25)$ \\
\hline Current & $44(35.6)$ & $1.43(0.60-3.38)$ & $1.10(0.20-6.11)$ \\
\hline
\end{tabular}


Table 3. Service factors in patients with pancreatic exocrine insufficiency, and results of adjusted univariable analysis of their association with initiation of PERT and clinical response

\begin{tabular}{|c|c|c|c|}
\hline Service factor & Patients with PEI & $\begin{array}{l}\text { Association with initiation } \\
\text { of PERT, OR }(95 \% \mathrm{CI})^{\mathrm{a}}\end{array}$ & $\begin{array}{l}\text { Association with } \\
\text { clinical response to } \\
\text { PERT, OR }(95 \% \mathrm{CI})^{\mathrm{a}}\end{array}$ \\
\hline FE1 result, $n=182$, mean (SD) & $92.9(93.2)$ & $1.00(0.99-1.00)$ & $1.00(0.99-1.01)$ \\
\hline FE1 repeated, $n=182, n(\%)$ & $42(23.1)$ & $3.06(1.38-6.77)^{b}$ & $0.50(0.14-1.75)$ \\
\hline Pancreatic imaging performed, $\mathrm{n}=182, \mathrm{n}(\%)$ & 149 (81.9) & $2.53(1.14-5.60)^{b}$ & $0.44(0.05-4.13)$ \\
\hline Abnormal pancreatic imaging, $n=143, n(\%)$ & $67(46.9)$ & $2.50(1.20-5.25)^{b}$ & $10.70(1.62-70.70)^{b}$ \\
\hline Nutritional blood tests performed, $\mathrm{n}=182, \mathrm{n}(\%)$ & $124(68.1)$ & $1.71(0.90-3.25)$ & $2.50(0.73-8.60)$ \\
\hline Abnormal nutrition blood tests, $\mathrm{n}=124, \mathrm{n}(\%)$ & $65(52.4)$ & $2.08(1.07-4.04)^{b}$ & $3.62(0.87-15.10)$ \\
\hline PERT prescribed, $\mathrm{n}=182, \mathrm{n}(\%)$ & $103(56.6)$ & $\mathrm{n} / \mathrm{a}$ & $\mathrm{n} / \mathrm{a}$ \\
\hline Starting dose of PERT, median (IQR), IU/meal & $50,000(0)$ & $\mathrm{n} / \mathrm{a}$ & $1.01(0.98-1.05)$ \\
\hline Dietitian referral, $\mathrm{n}=182, \mathrm{n}(\%$ ) & $77(42.3)$ & $\mathrm{n} / \mathrm{a}$ & $0.92(0.22-3.86)$ \\
\hline Followed up, $\mathrm{n}=182, \mathrm{n}$ ( \% ) & $99(54.4)$ & $\mathrm{n} / \mathrm{a}$ & $0.70(0.08-6.40)$ \\
\hline Clinical response to PERT, $n=81, n(\%)$ & $67(82.7)$ & $\mathrm{n} / \mathrm{a}$ & $\mathrm{n} / \mathrm{a}$ \\
\hline
\end{tabular}

Reference categories for alcohol excess and smoking are 'never'.

${ }^{a}=$ adjusted for age, sex and ethnicity; ${ }^{b}=p<0.05 ; C I=$ confidence interval; FE1 = faecal elastase- $1 ; G I=$ gastrointestinal; $I Q R=$ interquartile range; $n / a=$ not applicable; OR = odds ratio; PEI = pancreatic exocrine insufficiency; PERT = pancreatic enzyme replacement therapy; SD = standard deviation.

The adjusted univariable analysis found that patients with steatorrhoea, chronic pancreatitis, diabetes mellitus or previous alcohol excess were more likely to be started on PERT than those without each comorbidity (all $p<0.05$; Table 2 ). In addition, patients with a confirmatory low FE1 on repeat testing, abnormal pancreatic imaging or abnormal nutrition blood tests were more likely to be treated with PERT than patients with only one low FE1 or normal investigations (all $p<0.05$; Table 3 ).

Sixty-seven patients responded clinically to PERT ( $82.7 \%$ of patients whose response was documented). The associations with clinical response to PERT are shown in Tables 2 and 3. Clinical response to PERT was not associated with initial dose regimen (OR 1.01; 95\% CI 0.98-1.04) or referral to dietetics (OR 0.92; 95\% CI $0.22-3.86$ ). Patients with weight loss were more likely to respond to PERT than those without (OR 6.83; 95\% CI 1.23-37.93), and patients reporting flatulence were much less likely to respond to treatment (OR 0.04; 95\% CI 0.002-0.93). Patients with abnormal pancreatic imaging had 10 times the odds of responding to PERT than those with normal imaging (OR 10.70; 95\% CI 1.62-70.70). There was also a trend towards abnormal nutrition blood tests predicting response to PERT, however this did not achieve statistical significance (OR 3.62; 95\% CI 0.87-15.10).

There were no statistically significant differences in the management of patients with low FE1 between GI and non-GI clinicians, in terms of repeating FE1 or prescribing PERT (all p>0.05).

\section{Discussion}

While several international guidelines on the management of PEI are available, little is known about the clinical management of PEI in the UK. The practice of repeating FE1 and the predictors of clinical response to PERT have not been studied previously.

Twenty-eight per cent of our patients found to have a low initial FE1 result underwent repeat FE1 testing. Patients reporting bloating and flatulence were more likely to have their FE1 repeated, suggesting that clinicians may doubt the validity of FE1 results in patients without the classical symptoms of PEI. When a positive FE1 was repeated, the result was normal in almost half of patients, which contradicts the reported sensitivity and specificity of the assay. The initial FE1 value was the main predictor of a discordant repeat result, with normal repeat results found in $58.6 \%$ of patients with initial FE1 $100-199 \mu \mathrm{g} / \mathrm{g}, 50 \%$ of patients with initial FE1 $15-99 \mu \mathrm{g} / \mathrm{g}$ and $10 \%$ of patients with initial FE1 $<15 \mu \mathrm{g} / \mathrm{g}$. Beyond the initial FE1 value, we found very few differences between patients whose repeat FE1 results were concordant or discordant that could be used to predict which patients might benefit most from repeat testing. Therefore, we suggest the practice of confirmatory FE1 testing is extended to all patients whose initial FE1 is $15-199 \mu \mathrm{g} / \mathrm{g}$, particularly when pancreatic pathology is absent.

Most patients with PEI underwent pancreatic imaging, with computed tomography as the most commonly used modality. Approximately half of the imaged patients were found to have a pancreatic abnormality, with chronic pancreatitis diagnosed in one in four patients and pancreatobiliary malignancy in one in 20. This highlights the importance of imaging all patients with low FE1. A number of ultrasounds could not adequately view the pancreas; therefore, we propose that this modality be avoided for this indication.

We found abnormal nutritional parameters in half of the patients with PEI who had their nutritional biochemistry assessed. Objective measurement of malnutrition is vital in these patients, who are at risk of substantial morbidity as a result of malnutrition. ${ }^{12,13}$

Treatment with PERT could be positively identified in half of the patients diagnosed with PEI. There is no standardised dosing regimen at our hospital, but the majority of patients were commenced on 50,000 IU/meal of PERT, which is an appropriate 
initial dose. ${ }^{12,13}$ Most treated patients were offered follow-up and referred for formal dietetic input. However, a significant number of patients went untreated, and the rate of follow-up was low in these individuals.

It was not possible to ascertain the reasons for non-treatment in patients who did not start PERT. It is feasible that clinicians interpreted the FE1 as a false positive and elected not to start treatment; that treatment was offered but not documented; or that patients were lost to follow-up. Our analysis suggests that the former is the most likely explanation for non-treatment, given that the most well-established associated symptoms (steatorrhoea) and comorbidities (chronic pancreatitis, diabetes mellitus and alcohol excess) were predictive of being treated with PERT. In addition, our study suggests that repeating FE1, sending nutrition blood tests and imaging the pancreas can help guide therapeutic decision-making where the validity of a single low FE1 result is in doubt.

Of treated patients, $81.7 \%$ responded to PERT. The factors associated with achieving a satisfactory clinical response were previously unknown. We report that patients with abnormal pancreatic imaging were 10 times more likely to respond to PERT than patients with normal imaging. There was also a nonsignificant trend towards increased response-rate in patients with objective malnutrition. We therefore recommend a low threshold for repeating FE1 and investigating for other causes of malabsorption in patients with normal investigations who do not respond to PERT.

Although we did not have information on alcohol and smoking cessation interventions, it is notable that one-third of patients with PEI were current smokers, and one-fifth reported current alcohol excess. Alcohol and smoking cessation are recommended in the management of PEI, and should be part of a holistic management plan in these patients. ${ }^{12,13}$

\section{Strengths and limitations}

This study benefits from a large cohort of outpatients with low FE1 in an ethnically diverse region of the UK, with a wide variety of clinical symptoms and comorbidities. We included consecutive patients with a low FE1 result over a 6-year period, only excluding patients already prescribed PERT. As such, the study reflects everyday clinical practice, which should reduce selection bias. The statistical methods were robust and the variables of interest selected a priori. Potential confounders were identified and adjusted for.

However, all retrospective studies are vulnerable to information bias. Certain variables were based on clinical documentation, and the clinical differentiation of steatorrhoea from diarrhoea, for example, is difficult. Response to PERT was defined by subjective improvement of symptoms, and it would have been preferable to measure response using a more objective test, such as the CFA or $13 \mathrm{C}$ mixed triglyceride breath test. ${ }^{15}$ However, neither test is available in our centre. The use of serum biomarkers for assessing malnutrition was also suboptimal; particularly albumin, which is also a negative acute-phase reactant and reduced in hepatic disease. A comprehensive anthropometric assessment would have been more suitable but was not available for most patients.

We used FE1 $<200 \mu \mathrm{g} / \mathrm{g}$ to classify the presence of PEI. The FE1 assay shows high sensitivity and specificity for diagnosing severe PEI, but is less sensitive and specific than direct pancreatic function tests and the coefficient of faecal fat absorption in the diagnosis of mild and moderate PEI. ${ }^{4}$ We were unable to ascertain if any of the FE1 results were erroneous, but it is notable that almost half of the patients who did have their FE1 test repeated were re-classified as a result. Despite its limitations, FE1 is the only test for PEI available at most centres, therefore our study reflects the challenges in managing PEI in clinical practice. However, external validity has not been conducted, and it is conceivable that the generalisability of our results may be limited by local trends in clinical practice.

\section{Conclusion}

The management of PEI can, and should, be improved. False positive FE1 results are common, and the FE1 assay is insufficiently accurate in mild disease to advocate screening in low-risk populations. Clinicians might be reluctant to prescribe PERT after a single low FE1 result, therefore we recommend repeating FE1 routinely in patients with FE1 15-199 $\mu \mathrm{g} / \mathrm{g}$. Every patient diagnosed with PEI should undergo pancreatic imaging with computed tomography or magnetic resonance imaging, screening for malnutrition, dietetic assessment and be prescribed PERT at an initial dose $\geq 40,000 \mathrm{IU} / \mathrm{meal}$. As is the case with other chronic conditions, robust follow-up is essential.

\section{Summary}

\section{What is known?}

> Recent international guidelines on pancreatic exocrine insufficiency (PEI) recommend repeating faecal elastase-1 (FE1) in cases of diagnostic doubt; carrying out pancreatic imaging and nutrition blood testing; starting pancreatic enzyme replacement therapy (PERT); referring to a dietitian; and following up to monitor response.

\section{What is the question?}

> How does current clinical practice at a large UK centre compare to what is considered best practice?

$>$ Which factors predict repeating an FE1 $<200 \mu \mathrm{g} / \mathrm{g}$, the repeat FE1 being $\geq 200 \mu \mathrm{g} / \mathrm{g}$, initiation of PERT and clinical response to treatment?

\section{What was found?}

> A quarter of our patients with low (positive) FE1 underwent repeat testing, half of whom had a normal repeat result.

$>$ Patients with very low initial FE1 results $(<15 \mu \mathrm{g} / \mathrm{g})$ were unlikely to benefit from repeat testing.

> Patients with a confirmatory low FE1 on repeat testing were more likely to be started on PERT, as were patients with abnormal imaging or nutrition blood tests.

> Patients with abnormal pancreatic imaging were 10 times more likely to respond to PERT than those with normal imaging.

> Overall, treated patients were managed appropriately in terms of dosing regimen and referral to dietitians.

> However, treatment with PERT was documented in only half of patients with low FE1.

\section{What is the implication for practice now?}

> The initial management of PEI should adhere to the clinical standard described in Table 1, including pancreatic imaging in all patients with FE1 $<200 \mu \mathrm{g} / \mathrm{g}$

$>$ We also recommend repeat FE1 testing in patients with FE1 $15-199 \mu \mathrm{g} / \mathrm{g}$, particularly where there is diagnostic doubt. 


\section{Supplementary material}

Additional supplementary material may be found in the online version of this article at www.rcpjournals.org/clinmedicine: S1 - Detailed tables of results.

\section{References}

1 Singh VK, Haupt ME, Geller DE, Hall JA, Quintana Diez PM. Less common etiologies of exocrine pancreatic insufficiency. World ] Gastroenterol 2017;23:7059-76.

2 Arasaradnam RP, Brown S, Forbes A et al. Guidelines for the investigation of chronic diarrhoea in adults: British Society of Gastroenterology, 3rd edition. Gut 2018;67:1380-99.

3 Vanga RR, Tansel A, Sidiq S, El-Serag HB, Othman MO. Diagnostic performance of measurement of fecal elastase- 1 in detection of exocrine pancreatic insufficiency: systematic review and metaanalysis. Clin Gastroenterol Hepatol 2018;16:1220-8.e4.

4 Fischer B, Hoh S, Wehler M, Hahn EG, Schneider HT. Faecal elastase-1: Iyophilization of stool samples prevents false low results in diarrhoea. Scand J Gastroenterol 2001;36:771-4.

5 Pezzilli R, Morselli Labate AM, Ceciliato R et al. Quality of life in patients with chronic pancreatitis. Dig Liver Dis 2005;37:181-9.

6 de la Iglesia-García D, Huang W, Szatmary P et al; NIHR Pancreas Biomedical Research Unit Patient Advisory Group. Efficacy of pancreatic enzyme replacement therapy in chronic pancreatitis: systematic review and meta-analysis. Gut 2017;66:1354-5.

7 Campbell JA, Sanders DS, Francis KA et al. Should we investigate gastroenterology patients for pancreatic exocrine insufficiency? A dual centre UK study. J Gastrointestin Liver Dis 2016;25:303-9.

8 Roberts KJ, Schrem H, Hodson ] et al. Pancrease exocrine replacement therapy is associated with increased survival following pancreatoduodenectomy for periampullary malignancy. $H P B$ (Oxford) 2017;19:859-967.
9 Landers A, Muircroft W, Brown H. Pancreatic enzyme replacement therapy (PERT) for malabsorption in patients with metastatic pancreatic cancer. BMJ Support Palliat Care 2016;6:75-9.

10 Sikkens EC, Cahen DL, van Eijick C, Kuipers EJ, Bruno MJ. Patients with exocrine insufficiency due to pancreatitis are undertreated: a Dutch national survey. Pancreatology 2012;12:71-3.

11 Nikfarjam M, Wilson JS, Smith RC; Australasian Pancreatic Club Pancreatic Enzyme Replacement Therapy Guidelines Working Group. Diagnosis and management of pancreatic exocrine insufficiency. Med J Aust 2017;207:161-5.

12 Löhr JM, Dominguez-Munoz E, Rosendahl J et al. United European Gastroenterology evidence-based guidelines for the diagnosis and therapy of chronic pancreatitis (HaPanEU). United European Gastroenterol ] 2017;5:153-99.

13 Shandro BM, Nagarajah R, Poullis A. Challenges in the management of pancreatic exocrine insufficiency. World ] Gastrointest Pharmacol Ther 2018;9:39-46.

14 Lam KW, Leeds J. How to manage: patient with a low faecal elastase. Frontline Gastroenterology 2019:0:1-7.

15 Domínguez-Muñoz JE, Iglesias-García J, Vilariño-Insua M, Iglesias-Rey M. 13C-mixed triglyceride breath test to assess oral enzyme substitution therapy in patients with chronic pancreatitis. Clin Gastroenterol Hepatol 2007:5:484-8. [published correction appears in Clin Gastroenterol Hepatol 2011;9:1108].

16 Department of Health. UK Chief Medical Officers' alcohol guidelines review: Summary of the proposed new guidelines. London: Department of Health; 2016.

Address for correspondence: Dr Benjamin M Shandro, Research Fellow, Department of Gastroenterology, St George's University Hospitals NHS Foundation Trust, Blackshaw Road, London SW17 0QT, UK.

Email: bshandro@nhs.net 